\title{
Dispersion of Metallic Arc-discharged Single-walled Carbon Nanotubes by Two Polysilane Derivatives
}

\author{
Jinling Gao, ${ }^{1}$ Yongfu Lian, ${ }^{2}$ Chao Wen, ${ }^{1}$ and Hongmei $\mathrm{Bi}^{1,3 *}$ \\ ${ }^{1}$ College of Science, Heilongjiang Bayi Agricultural University, \\ Daqing 163319, China \\ ${ }^{2}$ Key Laboratory of Functional Inorganic Material Chemistry, Ministry of Education, \\ School of Chemistry and Materials Science, Heilongjiang University, Harbin 150081, China \\ ${ }^{3}$ College of Biological and Food Engineering, Guangdong University of Petrochemical Technology, \\ Maoming 525000, China
}

(Received May 26, 2019; accepted September 4, 2019)

Keywords: polysilane derivatives, selective dispersion, metallic single-walled carbon nanotubes, optical absorption spectrum, Raman spectroscopy

Two water-soluble polysilane derivatives were synthesized by the addition reaction of olefine acid with polysilane. After heating at $653 \mathrm{~K}$ and acidification, arc-discharged single-walled carbon nanotubes (SWNTs) were ultrasonically dispersed in aqueous solutions of two different polysilane derivatives and then subjected to ultracentrifugation to remove these insoluble species. Vis-NIR absorption spectra showed that the two polysilane derivatives have good dispersing ability toward metallic SWNTs (m-SWNTs). Moreover, the longer the side chain of the polysilane derivative, the higher the output of m-SWNTs. In addition, Raman spectra indicated that m-SWNTs with different chiral indexes are selectively dispersed in the two aqueous solutions of polysilane derivatives, indicating that the two polysilane derivatives can selectively identify m-SWNTs.

\section{Introduction}

Owing to the poor solubility of single-walled carbon nanotubes (SWNTs) and the existence of Van der Waals force between the SWNTs bundles, SWNTs are prone to aggregation. This restricts the promising applications of SWNTs in biomedicine, electrochemical biosensors, and chemical sensors. ${ }^{(1)}$ Therefore, it is very important to obtain highly dispersed SWNTs. Usually, solubilizers are used to increase the solubility of SWNTs in a solvent. ${ }^{(2)}$ If a solubilizer can recognize the electronic properties or structural characteristics of SWNTs, then the selective dispersion of SWNTs can be achieved. ${ }^{(3)}$ At present, the reported solubilizers include surfactants, ionic liquids, biomacromolecules, and polymers. ${ }^{(4)}$

In an organic solvent, SWNTs can be wrapped with some conjugated polymers. Owing to a large number of mobile electrons in conjugated polymers, a $\pi-\pi$ conjugate effect is generated between the conjugated polymer skeleton and the SWNTs, then the SWNTs are wrapped with

*Corresponding author: e-mail: hongmei_bi@126.com

https://doi.org/10.18494/SAM.2019.2446 
polymer segment groups. ${ }^{(5)}$ The side chains of conjugated polymers can be dissolved in an organic solvent, so SWNTs can effectively be dispersed in the organic solvent. SWNTs with different conductivities or diameters have been selectively dispersed by a series of polymers. SWNTs were reported to be dispersed by poly(4-vinylpyridine) and to form composites, then a glucose sensor was fabricated on the basis of these composites, and glucose was recognized within $3 \mathrm{~s}$ by the sensor. ${ }^{(6)}$ SWNTs wrapped with PEDOT derivatives have been used to design a sensor for chemical warfare agents. ${ }^{(7)}$ Yoon et al. ${ }^{(8)}$ reported that a precursor copolymer, $\mathrm{P}(4 \mathrm{VP}-\mathrm{VBAz})$, can disperse SWNTs in organic solvents, then a surface-immobilized $\mathrm{CO}_{2}$ sensor based on P(4VP-VBAz)-SWNT composites that showed high conductivity was developed. It was reported that polyfluorene-phenyl materials containing an electron-withdrawing side group can selectively disperse semiconductor SWNTs (s-SWNTs), whereas polyfluorene-phenyl materials containing an electron donor side group can selectively disperse metallic SWNTs (m-SWNTs). ${ }^{(9)}$ A very large number of s-SWNTs can be dispersed by an amphiphilic fluorenealt-pyridine conjugated copolymer with hydrophilic side chains; this dispersing solution was stable and suitable for transistor sensors. ${ }^{(10)}$ Naito et al. ${ }^{(11)}$ reported that CoMoCAT SWNTs can be dispersed by PMPS and PDHS, which have good flexibility, in an organic solvent, but PDBS with poor flexibility did not show dispersibility. Zhang et al. ${ }^{(12)}$ fabricated a new type of actuator sensor with polydimethylsiloxane-SWNT composites; this actuator showed great potential for use in smart windows, optical switches, and so on. Thus, the dispersal of SWNTs is important for sensing applications.

On the basis of the above ideas and the conclusions of our research reported previously, ${ }^{(13,14)}$ two water-soluble polysilane derivatives with different side chains, which were used to improve the solubility of SWNTs in water, were designed and synthesized, and the selective dispersive behavior of the two water-soluble polysilane derivatives toward SWNTs was studied from their optical absorption spectra and by Raman spectroscopy. The results show that the side-chain length of the polysilane derivative has a strong impact on the selective yield of m-SWNTs.

\section{Materials and Methods}

\subsection{Preparation of two water-soluble polysilane derivatives}

Two water-soluble polysilane derivatives were synthesized as shown in Fig. 1. Polysilane (PMS) was primarily synthesized by the Wurtz method, in which dichloromethylsilane was the monomer, sodium was the catalyst, and toluene was the solvent at a temperature of $383 \mathrm{~K} .{ }^{(15)}$ Then, PMS derivatives were synthesized by the hydrosilylation reaction in THF, in which 2,2'-azobisisobutyronitrile (AIBN) was used as the initiator in accordance with the literature. ${ }^{(16)}$ The specific process was as follows: $2.2 \mathrm{~g}(0.05 \mathrm{~mol})$ of PMS was dissolved in THF $(50 \mathrm{ml})$ in a glass flask, in which a small amount of AIBN was used to act as an initiator. The whole reaction process was continued for $8 \mathrm{~h}$ at $340 \mathrm{~K}$ under protective atmosphere of highly pure nitrogen $(99.99 \%)$, then $4.8 \mathrm{~g}(0.05 \mathrm{~mol})$ of 4-pentenoic acid was added drop by drop to the reaction system. After the reaction, the primary mixture was filtered with a vacuum suction filter. After the solvent (THF) was evaporated, the product was extracted with hexane several 


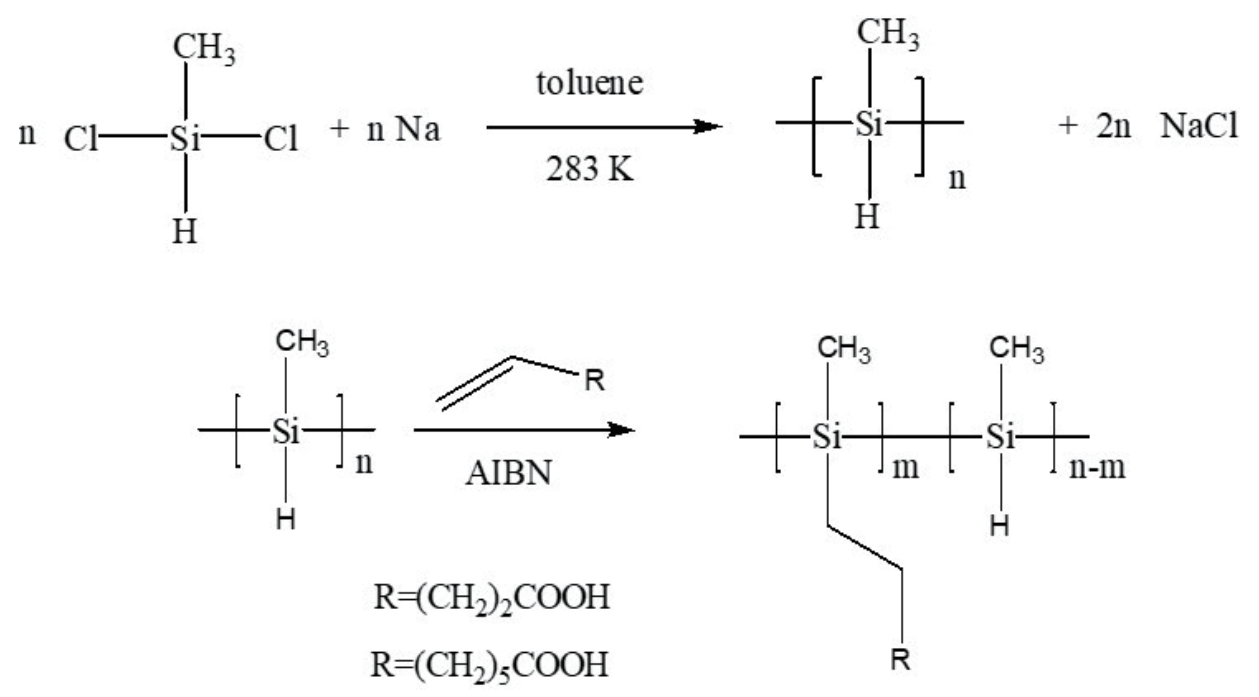

Fig. 1. Preparation of two water-soluble PMS derivatives.

times to remove the unreacted materials, and the purified product, polymethyl(5-pentenoic acidyl)silane (Pa-PMS), was obtained. The product was a thick yellow liquid that was soluble in toluene, THF, and alkaline water, partially soluble upon heating, but insoluble in n-hexane. In the same manner, polymethyl (8-octenoic acidyl) silane (Oa-PMS) was obtained.

\subsection{Synthesis and primary treatment of SWNTs}

Raw SWNTs were synthesized by the arc-discharge method. ${ }^{(17)} \mathrm{YNi}_{2}$ alloy and Ni were used as catalysts and FeS was used as the substance for growth. A specpure hollow graphite rod (with an external diameter of $\varnothing 8 \times 150 \mathrm{~mm}^{2}$ and an inner diameter of $\varnothing 6 \times 120 \mathrm{~mm}^{2}$ ) was used as the anode. The FeS:Ni:YNi2:C ratio was 1:1:3:5, and the powder was closely filled in the hole of the graphite rod. A graphite block $\left(40 \times 20 \mathrm{~mm}^{2}\right)$ was used as the cathode. The distance between the anode and the cathode was maintained at $0.5-1.0 \mathrm{~cm}$ during the discharge process, the electric current was $90 \mathrm{~A}$, and the discharge was performed under helium atmosphere of 450 Torr. After cooling, raw SWNTs were gathered from the top of the reaction chamber.

The raw SWNTs were placed in a porcelain crucible and then heated at $663 \mathrm{~K}$ for $2 \mathrm{~h}$ in air atmosphere, during which most of the amorphous carbon was burnt out. AC-SWNTs were thus obtained, and then the AC-SWNT samples were processed by the following two-step process. First, they were magnetically stirred with $12 \mathrm{~mol} / \mathrm{L} \mathrm{HCl}$ for $12 \mathrm{~h}$ at $330 \mathrm{~K}$, then filtered with a $0.22 \mu \mathrm{m}$ PTFE hydrophobic membrane to obtain HCl-treated SWNTs. Most of the metal catalysts were removed by adding HCl-treated SWNTs to a mixture of $\mathrm{H}_{2} \mathrm{SO}_{4} / \mathrm{HNO}_{3}$ (3:1) and allowing them to react for $15 \mathrm{~min}$. Finally, by filtration and washing with deionized water, $\mathrm{H}_{2} \mathrm{SO}_{4} / \mathrm{HNO}_{3}$-SWNTs were obtained. 


\subsection{Dispersion of SWNTs}

The PMS derivative Pa-PMS ( $1 \mathrm{~g})$ was placed in $100 \mathrm{ml}$ deionized water, then a small amount of $\mathrm{NaOH}$ was added to the solution until the $\mathrm{pH}$ of the solution was 8 . In accordance with a conventional process, $\mathrm{H}_{2} \mathrm{SO}_{4} / \mathrm{HNO}_{3}$-SWNTs $(5 \mathrm{mg})$ were ultrasonically dispersed in the solution for $24 \mathrm{~h}$ at a low temperature of $278 \mathrm{~K}$ to avoid the aggregation of $\mathrm{H}_{2} \mathrm{SO}_{4} / \mathrm{HNO}_{3}$ SWNTs. Then, the dispersion liquid was centrifuged with a high-speed centrifugal machine (CP70MX) at $15000 \mathrm{rpm}$ for $1 \mathrm{~h}$, and the upper $90 \%$ of the supernatant was carefully decanted. The solution of Pa-PMS-SWNTs was used to obtain vis-NIR and Raman spectra. A dispersion solution of Oa-PMS-SWNTs was acquired by the same method.

\subsection{Characterization methods}

The UV-vis-NIR optical adsorption spectra of SWNT samples were recorded by a vis-NIR spectrophotometer (UV3600, Shimadzu). Raman spectroscopy was performed at $633 \mathrm{~nm}$ $(1.96 \mathrm{eV})$ laser excitation with a microlaser Raman spectrometer (Renishaw in Via plus). The morphology of SWNT samples was observed by scanning electron microscopy (SEM) (S4800, Hitachi).

\section{Results and Discussion}

\subsection{Characterization of two PMS derivatives}

To confirm the formation of the two PMS derivatives, ${ }^{1} \mathrm{H}-\mathrm{NMR}$ spectra of PMS and two PMS derivative samples were recorded. Figure 2(a) shows the ${ }^{1} \mathrm{H}-\mathrm{NMR}$ spectrum of PaPMS and Fig. 2(b) shows the ${ }^{1} \mathrm{H}-\mathrm{NMR}$ spectrum of Oa-PMS. The characteristic peaks at 2.2 and $3.6 \mathrm{ppm}$ were caused by $\mathrm{CH}_{2}$ and $\mathrm{Si}-\mathrm{H}$, respectively. This showed that the peak intensity corresponding to $\mathrm{Si}-\mathrm{H}$ notably weakened, whereas that corresponding to $\mathrm{CH}_{2}$ notably

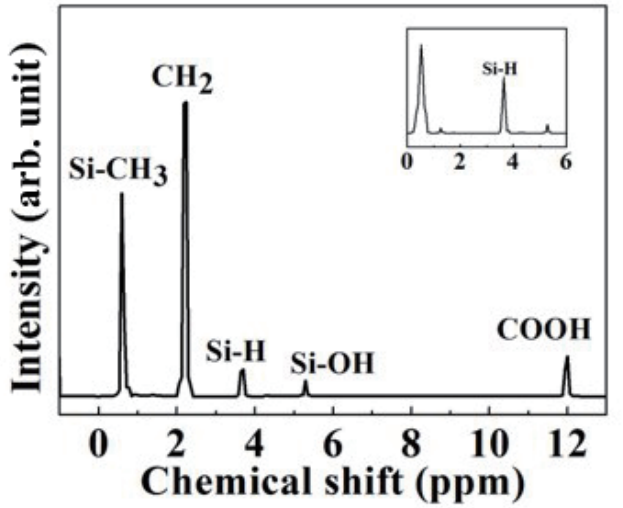

(a)

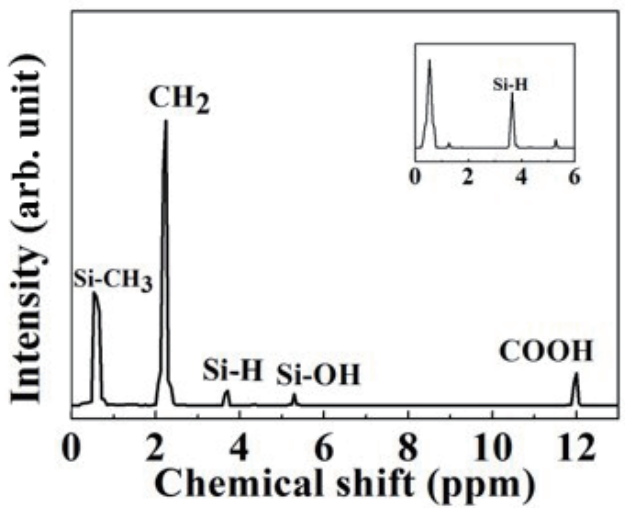

(b)

Fig. 2. $\quad{ }^{1} \mathrm{H}-\mathrm{NMR}$ spectra of (a) $\mathrm{Pa}-\mathrm{PMS}$ and (b) Oa-PMS. 
strengthened. Furthermore, characteristic peaks corresponding to $\mathrm{COOH}$ were observed at $12 \mathrm{ppm}$. This indicates that the anti-Markovnikov addition reaction occurred between the $\mathrm{Si}-\mathrm{H}$ bond and the $\mathrm{C}=\mathrm{C}$ bond, and that the side-chain functionalization of PMS was accomplished. Note that the solubility of the two PMS derivatives was improved in water because of the $\mathrm{COOH}$ groups in the side chains.

\subsection{Characterization of the dispersing behavior towards SWNTs of the two PMS derivatives}

The dispersing behavior of the two PMS derivatives towards SWNTs was evaluated by SEM, vis-NIR spectroscopy, and Raman spectroscopy. Shown in Fig. 3 are SEM images of AC-SWNTs, $\mathrm{H}_{2} \mathrm{SO}_{4} / \mathrm{HNO}_{3}$-SWNTs, Pa-PMS-SWNTs, and Oa-PMS-SWNTs. It is apparent that the $\mathrm{H}_{2} \mathrm{SO}_{4} / \mathrm{HNO}_{3}$-SWNTs have fewer residual particles than the AC-SWNTs and that the Pa-PMS-SWNTs and Oa-PMS-SWNTs have much cleaner surfaces and fewer residual particles than the $\mathrm{H}_{2} \mathrm{SO}_{4} / \mathrm{HNO}_{3}$-SWNTs. Thus, SEM observation evidences that the purity of SWNTs is markedly enhanced after the acidification and dispersing processes. Furthermore, this is a new approach for the purification of arc-discharged SWNTs.

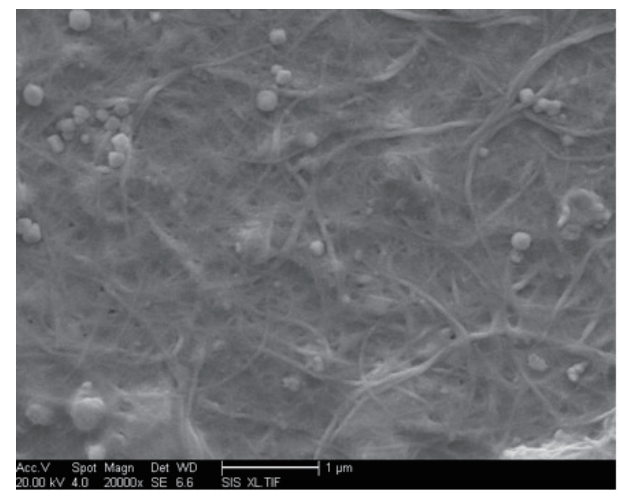

(a)

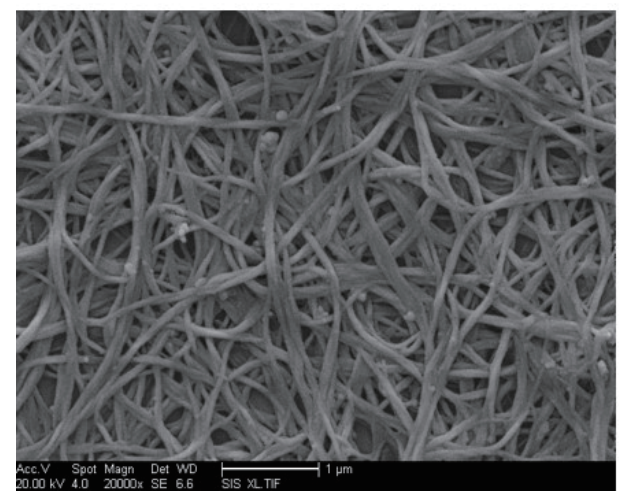

(c)

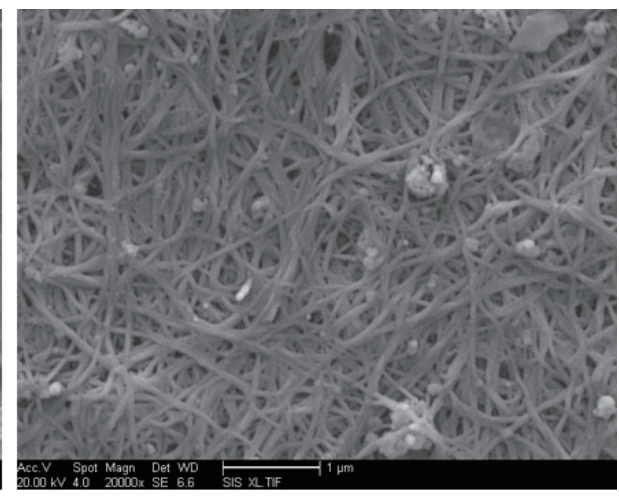

(b)

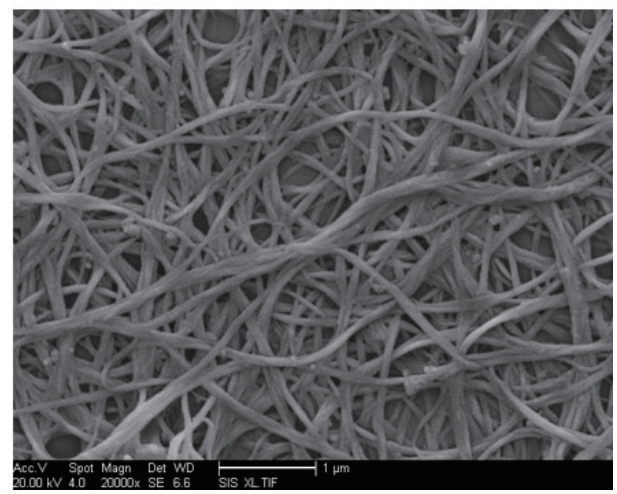

(d)

Fig. 3. SEM images of (a) AC-SWNTs, (b) $\mathrm{H}_{2} \mathrm{SO}_{4} / \mathrm{HNO}_{3}-\mathrm{SWNTs}$, (c) Pa-PMS-SWNTs, and (d) Oa-PMSSWNTs. 
Shown in Fig. 4 are the vis-NIR absorption spectra of $\mathrm{H}_{2} \mathrm{SO}_{4} / \mathrm{HNO}_{3}-\mathrm{SWNTs}$, Pa-PMSSWNTs, and Oa-PMS-SWNTs dispersed in water. For the arc-discharged SWNTs, the $\mathrm{S}_{33}$ and $\mathrm{S}_{44}$ absorption peaks at $400-600 \mathrm{~nm}$ are assigned to s-SWNTs, the $\mathrm{M}_{11}$ absorption peak at $600-800 \mathrm{~nm}$ is assigned to $\mathrm{m}-\mathrm{SWNTs}$, and the $\mathrm{S}_{22}$ absorption peak at $800-1200 \mathrm{~nm}$ is assigned to s-SWNTs. It was found that the absorption spectra of Pa-PMS-SWNTs and Oa-PMS-SWNTs have higher resolutions and absorption peaks than those of $\mathrm{H}_{2} \mathrm{SO}_{4} / \mathrm{HNO}_{3}$-SWNTs. In addition, compared with the absorption spectra of $\mathrm{H}_{2} \mathrm{SO}_{4} / \mathrm{HNO}_{3}$-SWNTs, the absorption spectra of PaPMS-SWNTs and Oa-PMS-SWNTs showed a blueshift, indicating the higher dispersing ability of Pa-PMS and Oa-PMS towards arc-discharged SWNTs.

In accordance with the method reported by Haddon et al. ${ }^{(18)}$ we compare the relative absorption intensities of m-SWNTs and s-SWNTs in Fig. 4. For $\mathrm{H}_{2} \mathrm{SO}_{4} / \mathrm{HNO}_{3}-\mathrm{SWNTs}$, the absorption peak area of $\mathrm{m}$-SWNTs relative to that of s-SWNTs was $0.193(0.021 / 0.109)$, whereas for Pa-PMS-SWNTs, the absorption peak area of m-SWNTs relative to that of s-SWNTs was 0.519 $(0.112 / 0.216)$. Therefore, compared with that of $\mathrm{H}_{2} \mathrm{SO}_{4} / \mathrm{HNO}_{3}$-SWNTs, the relative absorption peak area of Pa-PMS-m-SWNTs was 2.68-fold larger (0.519/0.193). This indicates that Pa-PMS had a selectively dispersive effect on m-SWNTs. In the same manner, for Oa-PMS-SWNTs, the absorption peak area of m-SWNTs relative to that of s-SWNTs was $0.528(0.121 / 0.229)$, and compared with $\mathrm{H}_{2} \mathrm{SO}_{4} / \mathrm{HNO}_{3}$-SWNTs, the relative absorption peak area of Oa-PMS-m-SWNTs was 2.74-fold larger (0.528/0.193). Thus, m-SWNTs can be selectively dispersed by both PaPMS and Oa-PMS. Furthermore, the longer the side chain of the PMS derivative, the greater the yield of m-SWNTs.

Raman spectroscopy is an important characterization method to ascertain carbon materials. In 1997, Rao et al. ${ }^{(19)}$ first reported the correlation between the excitation light energy and the Raman spectrum, which is derived from the Raman resonance scattering effect of SWNTs. Compared with that of monodisperse SWNTs, the radial breathing mode (RBM) of bunched

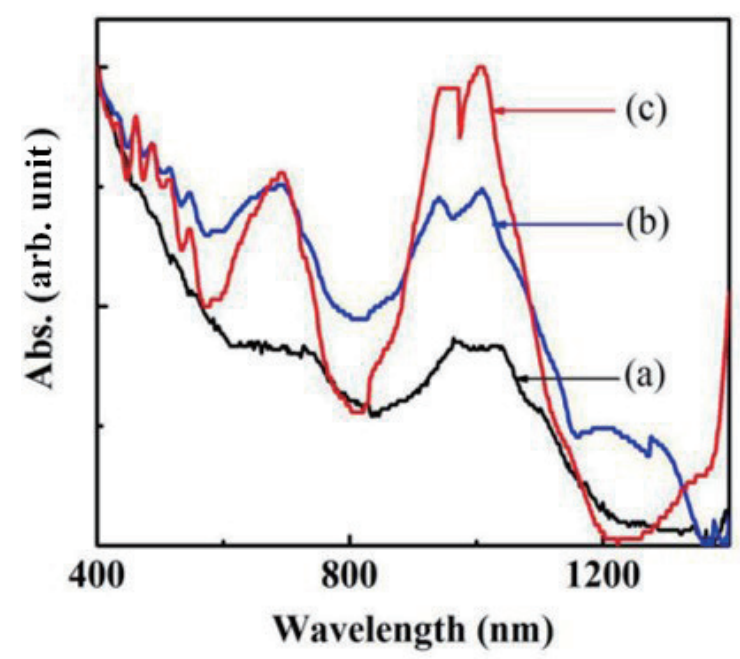

Fig. 4. (Color online) Normalized vis-NIR spectra of (a) $\mathrm{H}_{2} \mathrm{SO}_{4} / \mathrm{HNO}_{3}$-SWNTs, (b) Pa-PMS-SWNTs, and (c) Oa-PMS-SWNTs. 
SWNTs shifted $6-20 \mathrm{~cm}^{-1}$ in the low frequency direction, which is due to the Van der Waals force between bunched SWNTs. In 1998, Raman spectroscopy was first used to identify s-SWNTs and m-SWNTs. ${ }^{(20)}$ Accordingly, to further investigate the selective dispersion behavior of Pa-PMS and Oa-PMS towards SWNTs, Raman spectroscopy was performed on aqueous solutions of $\mathrm{H}_{2} \mathrm{SO}_{4} / \mathrm{HNO}_{3}$-SWNTs, Pa-PMS-SWNTs, and Oa-PMS-SWNTs.

Figure 5 shows the resonance Raman spectra of the solutions of $\mathrm{H}_{2} \mathrm{SO}_{4} / \mathrm{HNO}_{3}$-SWNTs, PaPMS-SWNTs, and Oa-PMS-SWNTs at $100-250 \mathrm{~cm}^{-1}$. The RBM peaks of Pa-PMS-SWNTs and Oa-PMS-SWNTs show a significant blueshift, indicating that after their dispersal by PaPMS and Oa-PMS, the tube bundles of SWNTs were effectively opened and the dispersal performance of SWNTs in the aqueous solutions was improved, which is consistent with the results obtained from the vis-NIR spectra above.

To further investigate the selective dispersion of Pa-PMS and Oa-PMS towards m-SWNTs, the RBM peak was processed into a sub-peak by origin at $100-250 \mathrm{~cm}^{-1}$. m-SWNTs or s-SWNTs with different chiral indexes were investigated by comparing the strengths of the RBM. The corresponding chiral index $(n, m)$ of the SWNTs obtained from the Kataura diagram ${ }^{(21)}$ is given in Fig. 5. The range of the RBM peaks of s-SWNTs is $126-170 \mathrm{~cm}^{-1}$ in Fig. 5 (a), and the range of the RBM peaks of m-SWNTs is $171-213 \mathrm{~cm}^{-1}$. There are five clear RBM peaks: s-SWNTs with chiral indexes of $(19,0)$ and $(18,2)$ were identified at $126-170 \mathrm{~cm}^{-1}$ and m-SWNTs with chiral indexes of $(15,3),(13,4)$, and $(14,2)$ were identified at $171-213 \mathrm{~cm}^{-1}$. The RBM peak area ratio of m-SWNTs to s-SWNTs was calculated to be 0.862 . In Fig. 5(b), the range of the RBM peaks of s-SWNTs is $141-182 \mathrm{~cm}^{-1}$ and the range of the RBM peaks of $\mathrm{m}$-SWNTs is $183-219 \mathrm{~cm}^{-1}$. There are three clear RBM peaks corresponding to m-SWNTs with a chiral index of $(13,4)$ and s-SWNTs with chiral indexes of $(19,0)$ and $(18,2)$. It was found by calculation that the RBM peak area ratio of m-SWNTs to s-SWNTs was increased to 1.339. The content of m-SWNTs was increased 1.55-fold (1.339/0.862) after the dispersion of SWNTs by Pa-PMS. A similar conclusion can be obtained from Fig. 5(c): the content of $\mathrm{m}$-SWNTs was increased 1.76-fold after the dispersion of SWNTs by Oa-PMS. Thus, the longer the side chain of the PMS derivative, the greater the yield of m-SWNTs, which was consistent with the results obtained from the vis-NIR spectra.

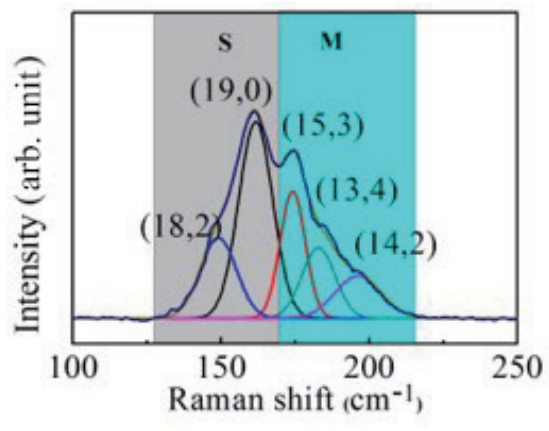

(a)

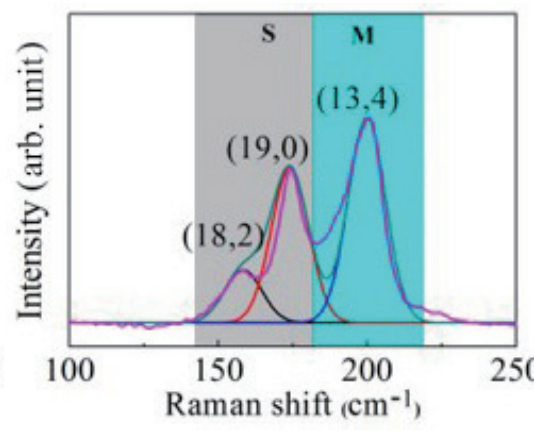

(b)

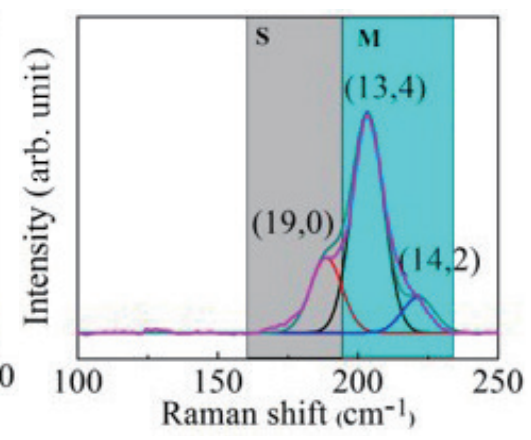

(c)

Fig. 5. (Color online) Raman spectra of (a) $\mathrm{H}_{2} \mathrm{SO}_{4} / \mathrm{HNO}_{3}-\mathrm{SWNTs}$, (b) Pa-PMS-SWNTs, and (c) Oa-PMS-SWNTs in the range of $100-250 \mathrm{~cm}^{-1}$. 


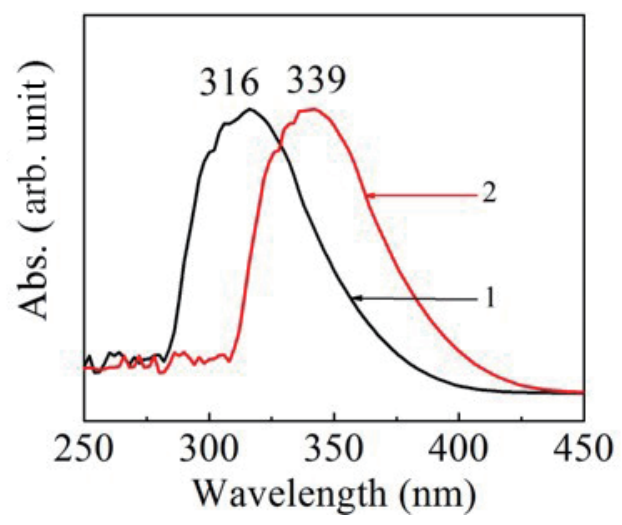

(a)

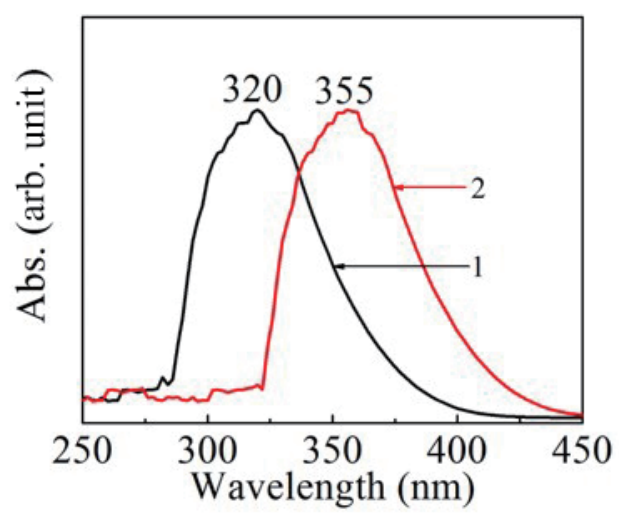

(b)

Fig. 6. (Color online) UV-vis spectra of (a-1) Pa-PMS, (a-2) Pa-PMS-SWNTs, (b-1) Oa-PMS, and (b-2) Oa-PMSSWNTs.

\subsection{Interaction between the two PMS derivatives and SWNTs}

The ultraviolet absorption of PMS is caused by the Si $-\mathrm{Si}^{*}$ bond. $^{(22)}$ The interaction between PMS derivatives and SWNTs can be illustrated by their UV-vis absorption spectra. Figure 6 shows the UV-vis absorption spectra of Pa-PMS and Oa-PMS before and after dispersing the SWNTs. In Fig. 6(a), curve 1 is the UV-vis absorption spectrum of Pa-PMS, whose maximum absorption wavelength is observed at $316 \mathrm{~nm}$, and curve 2 is that of Pa-PMSSWNTs, whose maximum absorption wavelength is observed at $339 \mathrm{~nm}$, showing a significant redshift. This illustrates that the transition of Si - Si $^{*}$ is changed because of the existence of SWNTs. This proves that the delocalized $\sigma$ electrons of Pa-PMS are adjacent to SWNTs in the process of dispersion, with a noncovalent $\sigma-\pi$ bond formed between Pa-PMS and the SWNTs. A similar conclusion can be reached from Fig. 6(b), which illustrates that a noncovalent $\sigma-\pi$ bond also formed between Oa-PMS and the SWNTs. Because the Fermi level of m-SWNTs is higher than that of s-SWNTs, the electron energy is relatively high, and it is easier to form a noncovalent $\sigma-\pi$ bond with PMS derivatives, so PMS derivatives have a higher dispersion ability towards m-SWNTs. Thus, the two water-soluble PMS derivatives tend to selectively disperse m-SWNTs. On the other hand, owing to the flexibility of the side chain of PMS, the longer the side chain of PMS, the easier the wrapping around SWNTs.

\section{Conclusions}

In this study, two water-soluble PMS derivatives were used to selectively disperse arcdischarged SWNTs, and stable dispersion solutions of SWNTs were obtained by ultrasonic dispersal at a low temperature. A series of characterizations showed that the dispersion solutions were enriched with $\mathrm{m}$-SWNTs. Thus, a method of enriching $\mathrm{m}$-SWNTs was proposed and applied. On the other hand, comparing m-SWNTs dispersed by the two PMS derivatives revealed that the longer the alkyl side chain of the PMS derivative, the greater the yield of 
m-SWNTs. Furthermore, charge transfer between the water-soluble PMS derivatives and the SWNTs was confirmed by UV-vis spectroscopy. The obtained disperse m-SWNTs have promising application in sensors.

\section{Acknowledgments}

This work was supported by the Talent Development Program in University (Grant No. ZRCPY201818), the National Natural Science Foundation of China (Grant No. 21503072), the Natural Science Foundation of Heilongjiang Province of China (Grant No. LH2019B012), the Start-up Program in University (Grant No. XDB201815), and the Project of Food Science Innovation Team of Guangdong Higher Education Institutes (2016KCXTD020).

\section{References}

1 V. Schroeder, S. Savagatrup, M. He, S. Lin, and T. M. Swager: Chem. Rev. 119 (2019) 1. https://doi. org/10.1021/acs.chemrev.8b00340

2 H. Sun, P. She, G. Lu, K. Xu, W. Zhang, and Z. Liu: J. Mater. Sci. 49 (2014) 20. https://doi.org/10.1007/s10853014-8436-4

3 H. Li, Q. Chen, and B. Han: New J. Chem. 40 (2016) 4. https://doi.org/10.1039/C5NJ03075G

4 N. Komatsu and F. Wang: Materials 3 (2010) 7. https://doi.org/10.3390/ma3073818

5 N. Nakashima and T. Shiraki: Langmuir 32 (2016) 47. https://doi.org/10.1021/acs.langmuir.6b02023

6 S. Soylemez, B. Yoon, L. Toppar, and T. M. Swager: ACS Sens. 2 (2017) 8. https://doi.org/10.1021/ acssensors.7b00323

7 J. F. Fennell, H. Hamaguchi, B. Yoon, and T. M. Swager: Sensors 17 (2017) 5. http://creativecommons.org/ licenses/by/4.0/

8 B. Yoon, S. Choi, T. M. Swager, and G. F. Walsh: ACS Appl. Mater. Interfaces 39 (2018) 10. https://doi. org/10.1021/acsami.8b11689

9 N. A. Rice, A. V. Subrahmanyam, B. Coleman, and A. Adronov: Macromolecules 48 (2015) 15. https://doi. org/10.1021/acs.macromol.5b00631

10 J. Ouyang, J. Ding, J. Lefebvre, Z. Li, C. Guo, A. J. Kell, and P. R. Malenfant: ACS Nano 12 (2018) 2. https:// doi.org/10.1021/acsnano.7b08818

11 M. Naito, K. Nobusawa, H. Onouchi, M. Nakamura, K. Yasui, A. Ikeda, and M. Fujiki: J. Am. Chem. Soc. 130 (2008) 49. https://doi.org/10.1021/ja806109z

12 W. Zhang, M. Weng, P. Zhou, L. Chen, Z. Huang, L. Zhang, and S. Fan: Carbon 116 (2017) 2. https://doi. org/10.1021/nn5048734

13 J. Gao, Y. Huang, and Y. Lian: RSC Adv. 5 (2015) 124. https://doi.org/10.1039/C5RA17761H

14 G. Liu, J. Gao, and Y. Lian: Fullerenes Nanotubes Carbon Nanostruct. 26 (2018) 9. https://doi.org/10.1080/1536 383X.2018.1440387

15 H. Kim and K. Matyjaszewski: J. Am. Chem. Soc. 110 (1988) 10. https://doi.org/10.1021/ja00218a064

16 L. Sacarescu, M. Simionescu, G. Sacarescu, and V. Hamciuc: J. Appl. Polym. Sci. 125 (2012) 2. https://doi. org/10.1002/app.35571

17 S. Iijima and T. Ichihashi: Nature 363 (1993) 6430. https://doi.org/10.1038/363603a0

18 M. E. Itkis, D. E. Perea, S. Niyogi, S. M. Rickard, M. A. Hamon, and R. C. Haddon: Nano Lett. 3 (2003) 3. https://doi.org/10.1021/n1025926e

19 A. M. Rao, E. Richter, S. Bandow, B. Chase, P. C. Eklund, K. A. Williams, and M. S. Dresselhaus: Science 275 (1997) 5297. https://doi.org/10.1126/science.275.5297.187

20 M. A. Pimenta, A. Marucci, S. A. Empedocles, M. G. Bawendi, E. B. Hanlon, A. M. Rao, and M. S. Dresselhaus: Phys. Rev. B 58 (1998) 24. https://doi.org/10.1103/PhysRevB.58.R16016

21 H. Kataura, Y. Kumazawa, Y. Maniwa, I. Umezu, S. Y. Suzuki, Y. Ohtsuka, and Y. Achiba: Synth. Met. 103 (1999) 1. https://doi.org/10.1016/S0379-6779(98)00278-1

22 J. Rabolt, D. C. Hofer, R. D. Miller, and G. N. Fickes: Macromolecules 19 (1986) 3. https://doi.org/10.1021/ ma00157a021 